\title{
Secondary opening of productive layers
}

\author{
Savenok Olga Vadimovna \\ Department of Oil and Gas Engineering named \\ after professor G.T. Vartumyan \\ Kuban state technological university \\ Krasnodar, Russia \\ olgasavenok@mail.ru \\ Petrushin Evgeniy Olegovich \\ Department of Oil and Gas Engineering named \\ after professor G.T. Vartumyan \\ Kuban state technological university \\ Krasnodar, Russia \\ eopetrushin@yahoo.com
}

\author{
Arutyunyan Ashot Straevich \\ Department of applied mathematics \\ Kuban state technological university \\ Krasnodar, Russia \\ mereniya@mail.ru
}

\author{
Likhacheva Olga Nikolaevna \\ Department of scientific and technical translation \\ Kuban state technological university \\ Krasnodar, Russia \\ olga-lihacheva@rambler.ru
}

\author{
Kusov Gennady Vladimirovich \\ Department of geophysical methods of search and investigation of mineral deposits \\ North-Caucasian Federal University \\ Stavropol, Russia \\ de france@mail.ru
}

\begin{abstract}
The article considers the influence of conditions in the well on the efficiency of layers' opening using perforation. The influence of the type of drilling muds and special liquids on the quality of secondary opening of productive layers was shown. The punched liquid was chosen during perforation and the punched environment of the weighed particles was cleaned. The technology of layers' opening by perforation in the environment of purified salt solution was described.
\end{abstract}

Keywords- drilling-in; quality of secondary drilling-in; punched liquid; cleaning of the punched environment; technology of drilling-in

\section{INFLUENCE OF CONDITIONS IN THE WELL ON EFFICIENCY OF DRILLING-IN USING PERFORATION}

The well having a punched face is called imperfect by nature of drilling-in. If the productive layer is opened with drilling not at full depth, then such well is called imperfect by the degree of drilling-in. In both cases, the actual output under other identical conditions will be less than the output of the well with an open stope because of emergence of additional filtration resistances [1].

If the well is imperfect by the degree and the nature of drilling-in, then the coefficient of hydrodynamic perfection will be defined by a formula:

$$
\varphi=\frac{\ln \left(\frac{R}{r}\right)}{\ln \left(\frac{R}{r}\right)+c_{1}+c_{2}},
$$

where $R$ - radius of the power loop; $r$ - borehole radius; $c_{1}$ and $c_{2}$ - the dimensionless coefficients considering additional filtration resistance because of imperfection of the well respectively to the degree and the nature of drilling-in.

The $c_{1}$ coefficient is defined by the extent of the productive layer opening, and coefficient $c_{2}$ depends on length $\ell_{p c}$ and diameter $d_{p c}$ of punched channels and density of perforation $n$. These coefficients can be found by V.I. Shchurov's schedules, constructed by results of experiments on models, or by the formulas obtained by mathematical processing of experimental data. At that, it is supposed that punched channels are ideal in geometrical and hydrodynamic sense, i.e. they have the correct cylindrical form, they are clean along the full length, there is no zone of the porous environment of the lowered permeability around them.

Let us estimate qualitatively the influence of parameters of perforation on the coefficient of hydrodynamic perfection of the well by formula (1), having accepted that the productive layer is opened with drilling through the entire thickness, i.e. $c_{1}=0$. Results of calculations have shown that at the density of perforation of 10 openings per $1 \mathrm{~m}$ and a greater increase in the depth of punched channels does not lead to significant 
growth of the perfection coefficient of the well. At very great values of density of perforation and length of channels, in this figure there is an area where the value of coefficient of perfection is more than a unit, i.e. a geometrically imperfect well has a higher productive characteristic in this area than if it was perfect. Under field conditions, there are practically no such cases [2].

The conducted experiments allow one to draw the following conclusion: the existing technology of perforation in overbalanced conditions with use of boring solutions with a firm phase exerts especially negative impact when drilling high-porous strata. The technology of drilling-in in overbalanced conditions and with use of water-based solutions with a firm phase leads to the fact that low-permeability strata become heavily soiled during drilling, and high-permeability ones - during perforation.

It is possible to prevent (or to lower) pollution of rock during perforation in overbalanced conditions taking the following events:

- application of mineralized (for example, stratal) or fresh water, improved by additive of surfactants, as a stirring solution;

- application of oil-based solutions;

- application of high-concentrated solutions of salts.

The characteristic of the offered stirring solutions is absence of the solid phase in them, which extremely reduces hydrodynamic efficiency of punched channels.

The surfactants introduced into punched liquid have to meet the following requirements:

- in small concentration - to considerably reduce a superficial tension at the interface «water - the hydrocarbon environment»;

- to improve wettability of rock with oil in the presence of water filtrate;

- not to form an insoluble deposit when in contact with the reservoir waters, salts and rocks which are contained in them;

- to inhibit dispersion and swelling of clay particles which are contained in reservoir rock in the presence of a water filtrate;

- to have a low indicator of adsorption on the pore space surface;

- to inhibit formation of adsorptive layers of gel structure at the interface.

For processing of punched liquid, both water-soluble and oil-soluble surfactants can be used. Water-soluble surfactants effectively reduce the superficial tension and the regional angle of wetting, facilitate the increase of relative permeabilities of the porous environment for oil and water and total permeability for them. Oil-soluble surfactants reduce effectively relative permeability of the porous environment for water; facilitate the reduction of water saturation of rock and thickness of hydrate covers, hydrophobize the surface of steam channels.

Nonionic surfactants are the most suitable for processing of punched liquids of water-based solutions as they are easily soluble in the fresh and mineralized water, little adsorbed on the surface of rocks, and effectively reduce superficial tension at the interface "water - oil" with low concentration (0.1$0.3 \%$ ). Ionogenic surfactants can leave sediments with the mineralized water; they are more intensively adsorbed on the water surface.

When using boring oil-based solutions, the perforation should also be carried out with application of similar stirring solutions.

The considered solutions can perform successfully functions of the stirring solutions only when the value of reservoir pressure is lower than hydrostatic. At reservoir pressures, which are above hydrostatic, the water solution of chloride calcium, which density can be brought to $1,40 \mathrm{~g} / \mathrm{cm}^{3}$, can be applied as homogeneous (without firm phase) punched liquid. It should be noted that the solution of chloride calcium facilitates aggregation of clay particles in the stratum; therefore negative consequences of application of water-based boring (clay) solutions during drilling decrease [3].

The cardinal direction of improving the technology of drilling-in is perforation in overbalanced conditions with a sealed wellhead, which can be carried out in two versions:

1) with the help of perforators, lowered into the well on the cable inside the tubing string;

2) with the help of perforators, descended into the well on a string of tubing.

Perforation in overbalanced conditions is a progressive way of secondary drilling-in, because at the time of creation of perforation channels under the influence of large pressure gradients, there is an intensive inflow of oil or gas from the stratum into the well, as a result of which self-cleaning of perforation channels and bottomhole formation occurs. In addition, for gas and oil fountain wells, the process of secondary drilling-in is combined with the process of causing an inflow of oil or gas from the stratum into the well.

Let us consider the technology and technique of perforation in depression. In the first variant, a perforator of the KPRU65, PR54, PR43 type is used. Before the perforator is lowered, the well is equipped with a tubing string, and a Christmas tree is installed at the mouth. A lubricator is installed in the place of the buffer branch - a device that allows lowering any devices into the working well in the presence of pressure on the mouth.

Reducing the level of the solution in the well (replacing it with a light solution, completely removing the solution from the well and filling it with air, natural gas, or nitrogen) creates the necessary difference between reservoir and bottomhole pressures chosen for these geological and technical conditions. In the well, through the lubricator of the necessary length (the maximum number of simultaneously discharged cumulative charges of the perforators should not exceed 150-300), a small 
punch is lowered on the wireline cable with its installation against the perforated interval. After the operation of the perforator, the stratum begins to manifest itself immediately, and an intensive process of cleaning the perforation channels and reservoir rock around the well occurs. In highly productive oil and especially gas producing wells, during filling wellbore with formation fluid, there is an intense increase in pressure at the mouth. The device of the lubricator is such that it makes it possible to withdraw the logging cable from the well, and if necessary, it can be re-launched into the well for the required interval [4].

When using small-sized perforators, a cumulative jet has to travel a long distance before colliding with an obstacle casing string, and it is known that the penetration depth in the barrier depends to a large extent on the thickness of the liquid layer. Therefore, the greatest effect should be expected from the use of such perforators in a gaseous medium. The experience of industrial application of this method of perforation has shown its high efficiency.

It is known that the greatest negative effect of the filtrate of drilling muds, penetrating into the stratum during drilling and perforation, is manifested when the gas-bearing strata are opened. Therefore, in the first place, it is necessary to provide for drilling-in through the tubing in the gas environment.

For drilling-in in overbalanced conditions by the second variant, using perforators descended by the tubing, hollow expendable guns, which operate not from the electric impulse (the cable is not present here), but from the impact mechanism. The latter works when one presses the rubber ball dropped onto the tubing string with the injection of liquid in them. Such perforators descend together with a special head equipped with a shock-explosive device for triggering accumulative charges. In these punchers, there are devices for transferring detonation from the section, which allows them to be screwed together to simultaneously open a formation interval of $50 \mathrm{~m}$ or more in thickness. After the operation of the perforator and the connection of the well to the formation, the perforated gun body remains in the well, if the well operates in a fountain manner.

The operation is reduced to the following. In the well, filled with drilling mud, the tubing string is lowered, at the bottom of which a perforator is mounted, with its installation against the opened interval. The wellhead is tied with a fountain valve to the required pressure. By removing a part of the liquid from the well by direct or reverse circulation or by replacing it with an easier one, the necessary underbalance is created, usually not exceeding $10 \mathrm{MPa}$; the bottomhole pressure must be not less than $5 \mathrm{MPa}$. A rubber ball is dropped through the wellhead bolt inside the tubing string, which is pushed through the flow of liquid through the pipes to a mechanism of impact-inclined action. The charge initiation device is activated. After connecting the stratum with the well, oil or gas from the stratum enter the tubing string both through the openings in the casing, formed after the triggering of the charges, and through special circulation windows, located above the perforator.
Puncher-type perforators are the only ones for which a logging cable is not used in the well and all work can be performed using the equipment available on the well.

The puncher is suitable for use in wells with a large angle of inclination, where it is difficult to lower the perforators on the cable, in the wells where perforation using pressure drawdown is expedient and the use of RP perforators is dangerous because of the fragments caused by the perforator (especially in the absence of a sump in the borehole). It is also used when drilling-in in wells of multicolumn structures, when the increased breakdown capacity of charges is necessary.

The disadvantages of this method of perforation include the inability to launch geophysical instruments (flowmeters, thermometers, etc.) into the working seam zone. This method does not allow re-perforation without lifting the tubing string, and, therefore, without damping the well with the solution.

\section{THE INFLUENCE OF THE TYPE OF DRILLING MUD AND} SPECIAL LIQUIDS ON THE QUALITY OF SECONDARY DRILLING-IN

In the Russian Federation, more than $90 \%$ of amounts of works on secondary drilling-in are carried out by cumulative perforation in the conditions when the bottom-hole pressure exceeds the formation pressure. At the same time, according to the existing integrated technical rules, while conducting drilling operations it is required to fill an operational column with the drilling mud fluid applied during initial drilling-in.

Abroad in a number of areas, some countries refused to carry out perforation works in the drilling mud environment, and for these purposes, they use special liquids for perforation without the solid phase or those that contain acid-soluble fillers.

In domestic practice, similar ways of conducting works have not been widely used. However, they are obligatory. Before the perforation in the overbalanced conditions, the casing shoe of the oil-well tubing or the oil-well tubing with a perforator is lowered. It is lowered to such depth, by which reliability of liquid replacement in the range of perforation and by $100-150 \mathrm{~m}$ above it is provided on a perforation liquid (invert emulsion, oil, diesel fuel, oil-based solution, water solution of sulphanole, polymeric solution, water solutions of chlorides and bromides $\mathrm{Na}, \mathrm{K}, \mathrm{Sa}, \mathrm{Zn}$, etc., stratum water). Then the perforator or the shoe is set in the necessary position and drawdown is produced (well liquid is replaced with oil, diesel fuel, foam, service water, lightened solutions without solid phase). For the perforation in the overbalanced conditions, the well (or a zone of the interval of perforation and by 100-150 m higher than it) should be filled with the perforation liquid which does not contain the solid phase. Optimum conditions of perforation during drawdown are provided by perforation of hydrocarbon-based liquids (oil, condensate, diesel fuel, invert emulsion, bitumen-lime mud). These liquids have to be compatible with formation fluids. Maintaining reservoir properties of productive strata is noted when using reservoir waters and water solutions of chloride calcium, chloride potassium, bromic potassium, bromic zinc as liquids of perforation [5]. 
The general requirements for all perforation and special liquids of hydro sanding perforation must be the following:

- they must be compatible to formation fluids and not to cause swelling of clays, deposition and emulsion formation;

- they must be technological from the viewpoint of preparation, storage, use;

- they must be compatible with the drilling mud fluid or liquids, filling the well;

- they must provide access of perforators to a perforation interval;

- corrosion activity of liquids should not exceed an admissible size;

- they should not pollute the environment;

- liquids and conditions of their application have to meet the requirements of fire and explosion safety and human safety.

The choice of perforation liquid is carried out depending on the category of productive rocks, physical properties of formation fluids, the size of formation pressure and the type of the drilling mud fluid applied during the initial drilling-in of productive rocks. For the right choice of a perforation liquid, it is necessary to conduct laboratory researches on compatibility of perforation liquid with the formation rock, the filtrate of drilling mud fluid sating it and formation fluids. At any category of rocks and any stratum pressure, if productive strata were drilled with the use of hydro carbonic drilling solutions (bitumen lime, invert, emulsion), only hydro carbonic liquids without the solid phase have to be used as perforation environment. If there is a need of weighting perforation liquids, then they should be made heavier with easily soluble weighting compounds $\left(\mathrm{CaCO}_{3}, \mathrm{FeCO}_{3}\right)$. Under normal and abnormally high formation pressures, if productive rocks were opened with water-based solutions, then it is necessary to apply water solutions of salts not containing the solid phase as perforation environments, which mineralization has to be not less than the mineralization of a filtrate of drilling mud fluid. If density of the chosen water solution of salts does not provide sufficient bottom-hole pressure, then the drilling mud fluid is pumped above the perforation interval, which is applied during initial drilling with a dividing buffer pack.

The technology of secondary formation opening by cumulative perforation passed three stages of development at present.

At the first stage, cumulative perforation was carried out in the drilling mud fluid. Data of domestic and foreign researches demonstrate that in these conditions there is mudding with clay particles of perforation channels; therefore their capacity decreases twice and more times. Unfortunately, such technology is applied on many deposits now.

The second stage of development is characterized by the use of special liquids without the solid phase as the perforation environment. Among such liquids, water solutions of salts, polymeric salt solutions, solutions on a hydro carbonic basis and some others are widely used ones [6].

The use of special liquids during secondary drilling-in gives higher effect in comparison with perforation in the environment of drilling (clay) mud fluid. However, at the same time the reservoir mudding by the weighed particles is not excluded in the course of its preparation, transportation and pumping to the well (fig. 1). Therefore, further improvement of technology of secondary drilling-in has demanded the solution of problems of deep purification of perforation liquids of the weighed particles. As a result the technology of secondary opening (the third stage) which distinctive feature is carrying out an additional complex of works on the decrease of the concentration of the weighed particles in the perforation environment has been widely used in foreign practice. The new technology provides the replacement of drilling mud fluid in the well with perforation liquids without the solid phase in several stages:

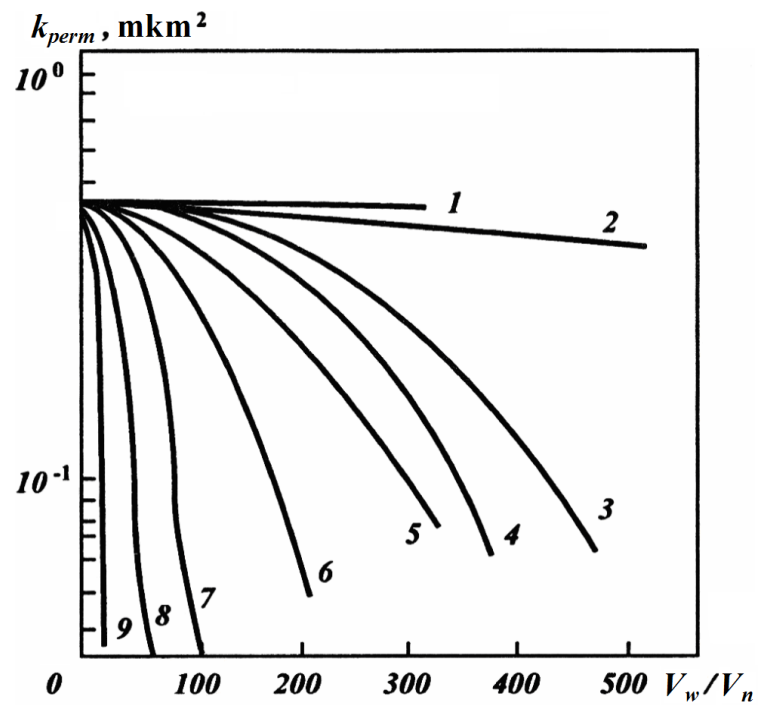

Figure 1 - Decrease in permeability of a core as a result of filtration of water with various concentration of the solid phase depending on the ratio of water volume $V_{w}$ to volume $V_{n}$ of the core pore space

\begin{tabular}{|l|c|c|c|}
\hline Number in figure & 1 & 2 & 3 \\
\hline Concentration of the solid phase, $\mathrm{mg} / \mathrm{l}$ & 2 & 2.5 & 14 \\
\hline Number in figure & 4 & 5 & 6 \\
\hline Concentration of the solid phase, $\mathrm{mg} / \mathrm{l}$ & 26 & 48 & 50 \\
\hline Number in figure & 7 & 8 & 9 \\
\hline Concentration of the solid phase, $\mathrm{mg} / \mathrm{l}$ & 84 & 110 & 485 \\
\hline
\end{tabular}

- replacement of drilling mud fluid with water in an operational column;

- washing a trunk of the well from the remains of drilling mud fluid by circulation of water with adding of alcohols and surface active substance in the closed cycle «capacity - the pump - the filter», and to remove the washed-away solid particles, «the well - capacity» cycle is used;

- replacement of water with the filtered perforation liquid. 
For the removal of the washed-away solid particles and perforation liquids from the water, filters of various designs are used, for example, net-shaped filters with the filtering elements in the form of plates, filled with quartz sand, etc.

The other important question defining a condition of the bottom-hole formation zone during drilling-in by perforation is the choice of the type of special liquids for definite geological conditions.

The degree of filling of an operational column with perforation liquid has an important practical value when determining expenses of time and funds for work on secondary drilling-in. By this criterion, two famous technologies of secondary drilling-in are distinguished.

The first one provides filling the whole trunk of the well with a special liquid. For its realization, it is necessary to prepare up to $50-60 \mathrm{~m}^{3}$ of perforation liquid per well operation. The considerable expenses connected with preparation, transportation, storage or utilization of large volumes of perforation liquid constrain the wide use of this technology in domestic practice [7].

The second perspective technology provides pumping a portion of perforation liquid to a perforation zone. Such technology implies that the perforation liquid fills, as a rule, only 100-300 m of the lower part of the trunk of the well. For creation of necessary overbalance in the drilled-in productive strata, the top part of an operational column is filled with drilling mud fluid or other liquid of the corresponding density. Due to the repeated reduction of the volume of the used perforation liquid, the costs of realization of this technology are much lower in comparison with the first one. However, during portion pumping, the special liquid becomes soiled and mixed up with the drilling mud fluid, filling the well. The last circumstance nullifies expediency of this liquid use. Therefore, the technology of secondary drilling-in with portion pumping of the liquid of special density to the zone of perforation demands the use of buffer dividers. They have to prevent the mixing of the perforation environment with drilling mud fluid within several days during repeated descents of perforators, geophysical devices, etc. to the well. They should not worsen its properties as a result of pollution by their own components when in contact with a perforation liquid.

\section{THE CHOICE OF PERFORATING LIQUID DURING PERFORATION}

When choosing perforating liquid for filling the perforation zone, it is necessary to be guided by the rules defining requirements for a filtrate of drilling mud fluid at a stage of initial drilling-in, and by the need to consider properties of the filtrate which penetrates into the stratum in the course of initial drilling-in. The new technique includes the following operations:

1) determination of initial oil permeability of core $k_{0}$ by established pressure difference $\Delta p_{0}$ during filtration of oil with constant consumption;
2) modeling of a stage of initial drilling-in (processing of the core with filtrate of drilling mud fluid in the amount corresponding to not less than five volumes of pore space);

3) modeling of a stage of secondary drilling-in (processing of the core with the perforating liquid in the amount corresponding to not less than five volumes of pore space);

4) modeling of the initial stage of development (replacement of perforating liquid from a core in the opposite direction a filtrate of drilling mud fluid);

5) modeling of a final stage of development (replacement of a filtrate of drilling mud fluid by oil before obtaining constant pressure difference by which final oil permeability of core $k_{x}$ and coefficient $\beta=k_{1} / k_{0}$ are determined.

In the course of replacement of filtrate, change of relative pressure difference on a core $\left(\Delta p_{m} / \Delta p_{0}\right)$ depending on the ratio of the volume of the filtered liquids to pore space volume $\left(V_{\text {liquid }} / V_{\text {rock }}\right)$ is registered. Function $\Delta p_{m} / \Delta p_{0}=f\left(V_{\text {liquid }} / V_{\text {rock }}\right)$ allows estimating qualitatively the value of overbalances necessary to cause the inflow of stratum fluid, i.e. the degree of complexity of well development.

Using this technique, the influence of the main water- and hydrocarbonic-based perforating liquids on a collector is investigated. Results of experiments have shown that the efficiency of using special liquids substantially depends on conditions of initial drilling-in of strata [8].

Experiments have shown that with other things being equal, the minimum pollution of a collector is reached in case of use during initial drilling-in and perforation of solutions with the similar nature of wetting. Comparison of efficiency of the use of water-based various special liquids during perforation shows that polymeric solutions concede to pure salt solutions both by the degree of complexity of replacement of filtrates and by the value of coefficient $\beta$.

Therefore, in the conditions of initial autopsy of layers with use of water solutions, it is necessary to include solutions of salts, various on density in the number of the most perspective punched liquids $\mathrm{Na}^{+}, \mathrm{K}^{+}$и $\mathrm{Ca}^{2+}$. For preparation of salt solution with a density of up to $1,40 \mathrm{~g} / \mathrm{cm}^{3}$, it is expedient to use calcium chloride, and for receiving heavier - calcium bromide.

During realization of high-economic option of technology of secondary drilling-in with portion pumping of the perforating liquid in a perforation zone, one of the main issues is the choice of a buffer divider. In these conditions, the buffer has to prevent the mixing of the perforating medium and drilling mud fluid both in the course of pumping to the well and within the next several days during repeated descents of punchers, geophysical devices, etc. This task is difficult as it is connected with the need to implement mutually exclusive requirements. On the one hand, for reliable dissociation of systems, it is necessary to create a strong structure in a buffer divider; on the other - indicators of structural properties of buffer solution have to be such that free passing of the puncher was provided. 
When using water solutions as buffer dividers, separation of punched liquid and drilling mud fluid in the course of their consecutive flowing in the column is possible. However at the same time, considerable pollution of liquid by polymers is noted, which negatively influences filtration properties of the collector. To prevent this process and to reliably isolate the liquid are possible when using invert emulsion as a buffer divider. In this case, buffer liquid is opposite by wetting nature to the separated liquids. Mutual dissolution of liquids does not happen, which prevents pollution of special liquids with components of a buffer divider.

Therefore, it is possible to recommend the use invert emulsions with the increased content of water component up to $60-70 \%$ as buffer liquid for isolation of a portion of the salt solution during secondary drilling-in. The necessary heat stability of such systems is reached due to the choice of the corresponding type of emulsifier. At bottomhole temperatures of up to $90{ }^{\circ} \mathrm{C}$, emultal, widely used in oil industry, can be applied as the latter. The density of invert emulsions can be regulated by using salt solutions of necessary concentration as a water component. The increase of the density of the emulsion by more than $1,20 \mathrm{~g} / \mathrm{cm}^{3}$ is reached due to application of solid weighting compounds, for example, of chalk or barite [9].

\section{CLEANING OF THE PERFORATING ENVIRONMENT FROM THE WEIGHED PARTICLES}

Without careful purification of the perforating liquid of the weighed particles, it is impossible to consider the solution of the problem of high-quality drilling-in. However, purification of the liquid with the use of filters is difficult and laborconsuming.

The technological scheme of cleaning a portion of perforating liquid (salt solution) in the well includes the following stages:

- processing of perforating liquid by flocculant on the surface;

- delivery of the liquid to a perforation zone with isolation from drilling mud fluid by a buffer divider;

- upholding the liquid on a face for precipitation of the weighed particles in a sump.

The method of upholding is widely applied in sewage treatment during complex processing by their coagulant and flocculant.

Optimum conditions for cleaning of the perforating environment with the method of upholding are reached during introduction cations of calcium and its treating by 0,005 $0,007 \%$ of Polyacrylamide in the composition of salt solution. The process of clarification of a column of salt solution $300 \mathrm{~m}$ high does not exceed preparatory work for perforation by the duration and, therefore, does not demand additional expenses of time.

Thus, with the developed technique of cleaning a portion of the salt solution on a well face, a high degree of removal of the weighed particles is reached, and additional delays in the course of secondary drilling-in are practically not created.

\section{TECHNOLOGY OF DRILLING-IN BY PERFORATION IN PURIFIED SALT SOLUTION}

The technology of secondary drilling-in, providing filling a zone of perforation with the water salt solution, containing cations of calcium and flocculant, its separation from drilling mud fluid in the portion of an invert emulsion, cleaning of the punched environment of the solid phase with an upholding method on a face and work on perforation of a column is developed. At a preparatory stage of realization of technology, types and volumes of liquids for filling of the well are chosen.

The drilling mud fluid applied at initial drilling-in is used as liquid for filling the top part of a trunk of the well. Such solution has to have good sedimentation stability for prevention of loss of a weighting compound and its accumulation at the interface of buffer liquid that can complicate the passing of punchers. Additional processing of drilling mud fluid to achieve the required indicators of technological properties is carried out before works on pumping of perforating liquid to the well. Below the drilling mud fluid, there is the portion of buffer liquid - a divider. Under the influence of gravitation, it is necessary that the density of the solutions, filling the well, increased in the direction from top to down by not less than $0,20-0,40 \mathrm{~g} / \mathrm{cm}^{3}$ for prevention of movements of liquids.

It is expedient to use salt solution of the same type which fills a perforation zone as a water component of an invert emulsion. Density of water component $\rho_{w c}$ is determined proceeding from the necessary density of buffer liquid $\rho_{b l}$ at a preset value of water content $W$ of an emulsion:

$$
\rho_{w c}=\frac{\rho_{b l}-\rho_{h c} \cdot(1-W)}{W},
$$

where $\rho_{h c}-$ density of a hydrocarbonic component of an invert emulsion.

The choice of values $W$ has to exclude or minimize whenever possible application of a weighting compound for achievement of the necessary density of buffer liquid. The volume of buffer liquid defines 100-150 m of a trunk of the well at the rate on filling.

Below a buffer divider, there is perforating liquid - the perforating environment. The salt solution applied as the perforating environment has to contain not less than $2 \mathrm{~g} / \mathrm{l}$ of cations of $\mathrm{Ca}^{2+}$ (coagulant) and $0,005-0,007 \%$ of Polyacrylamide (flocculant). The volume of a portion of salt solution is recommended to be determined using the rate on overlapping of the lower part of a trunk of the well to the level located by 50-100 m higher than the top punched openings.

Salt solution and buffer liquid are prepared in the clay mixer or in measured capacities of the cementing unit. In the latter case, for dissolution of salt, an additional tank with a capacity of $0,5-1,0 \mathrm{~m}^{3}$ is used. To prepare perforating liquid 
and an inert emulsion is better centrally, in special point, with delivery to the boring by means of tanker trucks. Processing of salt solution by flocculant (Polyacrylamide), as well as addition in need of a coagulant is carried out directly in the cementing unit capacity when hashing in the course of circulation on the closed cycle within 15-30 min.

The zone of perforation is filled with portion of salt solution after pressure testing of an operational column. For this purpose to the well lower a column of pump and compressor pipes to a face and tie her with the cementing unit. One measured capacity of the cementing unit is filled with a buffer divider, and another - salt solution [10].

Pumping liquids is based on the principle of balance of pressure in a column of pump and compressor pipes and annular space and is carried out in a certain sequence:

- buffer divider with volume $V_{1}$ providing the filling of ring space between the casing pipe and compressor pipes at preset height $H$;

- salt solution in design volume;

- buffer divider with volume $V_{2}$ sufficient for the filling of the casing pipe of the pump and compressor pipes in the range of height $H$;

- squeezing liquid (of the same type as the solution, which fills the well) in the amount providing delivery of perforating liquid to a perforation zone.

The volumes of the first and second portions of the buffer divider are calculated using formulas:

$$
\begin{gathered}
V_{1}=0,785 \cdot\left(D_{1}^{2}-D_{2}^{2}\right) \cdot H ; \\
V_{2}=0,785 \cdot D_{3}^{2} \cdot H,
\end{gathered}
$$

where $D_{1}$ - internal diameter of an operational pipe; $D_{2}$ and $D_{3}$ - respectively external and internal diameters of pump and compressor pipes.

In order to make necessary overbalance on the stratum, one needs heavier liquid. If in the well before pumping the perforating liquid, there was water, then in this case, after completion of driving, the casing of pump and compressor pipes is lifted to the upper bound of the buffer divider with the subsequent replacement of water with drilling mud fluid.

The minimum gap in time between pumping buffer liquid and the beginning of perforation is defined by duration of sedimentation of the weighed particles from a perforation zone in a sump. As a rule, this time is less than duration of raising the casing of pump and compressor pipes and installation of a punched latch on the mouth of the well.

Later, the works on perforation and development of the well are carried out according to the existing normative and technical documents.

\section{PERForation OF CEMENT STONE}

During perforation of casing pipes and a cement ring in the latter, there is tension both at the expense of a blast wave and due to passing of a bullet or a cumulative stream. The efficiency of perforation in the cement ring depends on many factors. With other things being equal, the depth of the punched channel depends on the density of a barrier, the degree of saturation by water, and its mechanical properties (strength, plasticity, the resilience to short-term loadings, compressibility etc.). However, the values of durability defined during static application of loading can not characterize completely the breed resilience to destruction as during explosion, the large role is played by dynamic loadings.

Inspection of an interval of perforation in wells and at stands by means of cameras and other methods has revealed existence of cracks of various sizes in a pipe body. In some cases, when washing wells after perforation, fragments of cement stone and breed are carried out. Deformation of cement stone is followed by its cracking. With the reduction of mechanical durability of samples during both bullet and cumulative perforation, the diameter of the opening increases (especially during cumulative perforation), and the surface of the channel becomes less equal.

With the increase of temperature up to $75^{\circ} \mathrm{C}$, channels had a more regular shape during both bullet and cumulative perforation, which is explained by the increase in mechanical durability of samples. There were no cracks.

At a temperature of $110{ }^{\circ} \mathrm{C}$ and pressure of $10 \mathrm{MPa}$ strength of samples on a bend changed from 2,72 in 3 hours up to $4,6 \mathrm{MPa}$ in 96 hours. With a pressure of $10 \mathrm{MPa}$ and temperature of $140{ }^{\circ} \mathrm{C}$ strength on a bend and compression decreased from 3,08 respectively and $6,42 \mathrm{MPa}$ in 3 hours to 1,65 and $3,85 \mathrm{MPa}$ in 96 hours. The samples hardening at more high temperatures differed by the fragile structure. During cumulative perforation, the samples hardening at a temperature of $110{ }^{\circ} \mathrm{C}$ had approximately identical openings and character of their surface. The samples hardening at higher temperatures $\left(140\right.$ and $\left.200{ }^{\circ} \mathrm{C}\right)$ and pressure of $10 \mathrm{MPa}$, because the cement stone has smaller durability, has strongly collapsed. And at the accepted sizes of samples, there was «blowing» of a cement stone. In all cases, no cracking was noted on samples or the escaped pieces. With the increase in the diameter of samples up to $200 \mathrm{~mm}$ during perforation, their results remained the same.

With the increase in mechanical durability of the cement stone, the depth of bullets' penetration during perforation of SSP-4 1/4" decreases. In the presence of a 10-mm steel plate, the bullet puncher can effectively punch a cement stone to the depth of $100 \mathrm{~mm}$ with a durability on a bend of 6,0-7,0 MPa. Due to the impurity of the productive horizons, it is almost difficult to obtain effective communication between them and the well by means of the specified puncher.

The Portland cement and slag stones, created in the well $(t$ $=140{ }^{\circ} \mathrm{C}, p=45.0 \mathrm{MPa}$ ) between concentric the located pipes at perforation, did not crack [11]. 
Cracking of a pipe in these zones was also not observed, except for only those cases when punched openings were located very closely to each other.

In the well where the drilling mud fluid is forced out incompletely and it (or the dehydrated clay crust) is between a cement stone and the well wall, the cement stone during perforation cracks and disintegrates, whereas during the continuous filling of the ring space, the cement stone is not divided into pieces.

\section{References}

[1] «Schlumberger Dowell» - Works and services with flexible tubing, Oil and Capital, № 1, pp. 77-78, 1998.

[2] G. Suman, R. Elis Cementing Handbook. Including Casing Handling Procedures, Reprinted from World Oil, Gulf Publishing, 1977.

[3] M.O. Ashrafyan, O.A. Lebedev, N.M. Sarkisov, Perfection of the design of the face of wells, M.: Nedra, 1987.

[4] R.S. Yaremichychuk, Yu.D. Kachmar, Opening of productive horizons and development of wells, Lviv, 1982.
[5] S.V. Falkovich, About the mechanism of opening of layers by abrasive hydro-sandblast perforation, Commercial geology, M., Issue. 21, pp. 213-230, 1969.

[6] A.I. Bulatov, O.V. Savenok, Completion of oil and gas wells: theory and practice, Krasnodar: LLC «Prosveshchenie - Yug», 2010, 539 p.

[7] A.I. Bulatov, O.V. Savenok, Complications and accidents in the construction of oil and gas wells, Krasnodar: LLC «Prosveshchenie Yug», 2010, $522 \mathrm{p}$.

[8] A.I. Bulatov, O.V. Savenok, R.S. Yaremiychuk, Scientific bases and practice of development of oil and gas wells, Krasnodar: LLC «Publishing House - South», 2016, 576 p.

[9] A.I. Bulatov, O.V. Savenok, Capital overhaul of oil and gas wells: 4 volumes, Krasnodar: LLC «Publishing House - South», 2012-2015, V. $1-4$.

[10] A.I. Bulatov, O.V. Savenok, Workshop on discipline «Completion of oil and gas wells»: in 4 volumes: textbook, Krasnodar: LLC «Publishing House - South», 2013-2014, V. 1-4.

[11] D.A. Berezovsky, O.V. Savenok, A.V. Lavrentiev, Development of physical and chemical models and methods for predicting the state of reservoir rocks, Oil Industry, No. 9, pp. 84-86, 2014. 